University of Nebraska - Lincoln DigitalCommons@University of Nebraska - Lincoln

CSE Conference and Workshop Papers

Computer Science and Engineering, Department of

$10-2014$

\title{
Safety Considerations for Small Unmanned Aerial Systems with Distributed Users
}

Brittany A. Duncan

University of Nebraska-Lincoln, bduncan@unl.edu

Robin R. Murphy

Texas Aઐ'M University,murphy@cse.tamu.edu

Follow this and additional works at: http://digitalcommons.unl.edu/cseconfwork

Duncan, Brittany A. and Murphy, Robin R., "Safety Considerations for Small Unmanned Aerial Systems with Distributed Users" (2014). CSE Conference and Workshop Papers. 239.

http://digitalcommons.unl.edu/cseconfwork/239

This Article is brought to you for free and open access by the Computer Science and Engineering, Department of at DigitalCommons@University of Nebraska - Lincoln. It has been accepted for inclusion in CSE Conference and Workshop Papers by an authorized administrator of

DigitalCommons@University of Nebraska - Lincoln. 


\title{
Safety Considerations for Small Unmanned Aerial Systems with Distributed Users
}

\author{
Brittany A. Duncan and Robin R. Murphy
}

\begin{abstract}
This paper identifies three categories of safety risks posed by allowing multiple users to engage with small Unmanned Aerial Systems (sUAS) and offers five recommendations on how to reduce or mitigate these vulnerabilities. Data from sUAS can benefit multiple experts at a disaster who may not be familiar with robots or colocated with the pilot. Two different styles of interfaces have been developed and tested with responders conducting exercises to facilitate team coordination with a quadrotor at Texas A\&M Engineering Extension Service's Disaster City__ over a four year period. The two interfaces illustrate three distinct categories of safety concerns: unsafe control regimes, loss of situation awareness, and increased stress. Five recommendations are proposed to mitigate or eliminate the safety concerns: separate the payload camera from the platform, giving the pilot a dedicated "pilot-cam" and the experts a fully gimbaled payload; use artificial intelligence to resolve conflicts between competing directives from multiple experts; allow the pilot, or a software agent, to turn off the expert's ability to control or communication; use multi-modal warnings rather than rely on visual cues; and add guarded motion to prevent collisions.
\end{abstract}

\section{INTRODUCTION}

Small Unmanned Aerial Systems (sUASs) are increasingly being used in response operations with 14 deployments reported in [1] through 2013. As noted by Murphy in the 2011 SSRR Outrageous Visions in Computing Paper Competition [2], a challenge for sUAS is that the real-time data from even a single sUAS will be of use to multiple, distributed users and each user will have their own competing priorities and mission needs. The ability for a tactical responder, a task force leader, an incident commander, or even an structural or medical expert to work through the internet with the sUAS is exciting.

In order to support distributed users, we have engaged in research on multi-modal user interfaces that support team coordination through shared visual common

This work was supported by an NSF Graduate Research Fellowship B.A. Duncan and R.R. Murphy are with the Center for Robot-Assisted Search and Rescue, Texas A\&M University, College Station, TX 77843-3112 bduncan@cse.tamu.edu, murphy@cse.tamu.edu ground. Peschel et al. [3] notes that based on humancomputer interaction principles, each user, which will be referred to as a Mission Specialist to distinguish the user from the Pilot as a user, will need a display tailored to for their needs. Working with expert responders from the Texas Engineering Extension Service, our center has designed a series of user interfaces over a four year period, and refined them by flying in over a dozen exercises at the Texas A\&M Engineering Extension Service's (TEEX) Disaster City ${ }^{\circledR}$ complex using an AirRobot 100B quadrotor. Two of the tablet interfaces were extremely popular with responders. One interface allowed a Mission Specialist to directly control the camera payload, reducing the degree of team coordination with the Pilot. The other interface allowed Mission Specialists and the Pilot to communicate through sketch, multi-touch, and audio links.

However, we have found that both user interfaces introduce subtle safety issues, depending on the platform and the users. Fortunately, these safety risks can be sufficiently mitigated or eliminated through platform changes and increased intelligent software. This paper reviews related work in sUAS distributed team coordination and multi-modal user interface design, then describes the two most popular user interfaces from our research. Next, the safety risks created by the two interfaces are identified, followed by recommendations on how to overcome the potential problems.

\section{RELATED WORK}

Peschel and Murphy [4] provides a survey of interfaces for users interacting with the UAS who are not the Pilot. The likelihood of multiple users beyond the operator engaged with a small UAV has been acknowledged by Gledhill et al [5] for wilderness search and rescue and by Cavett et al in general [6]. Pond et al [7] report on how visual and verbal communication interferes in UAS for the US Air Force, while Pratt et al. [8] discuss the construction of verbal communication protocols to ensure safety.

While sketch, multitouch, speech-based, and other multi-modal interfaces for small UAVs have been con- 
sidered in the literature, these works do not consider the impact of these communication modalities on overall system safety. Cummings et al [9] describe the use of sketch for planning UAS missions but did not discuss any possible negative consequences or how multiple users would use sketch. Guo et al [10] allowed users to cooperatively plan missions using multi-touch on a table surface interface, while Jourde el al [11] focuses on a design notation for multi-touch control for generating plans; neither consider vehicle safety. Speechbased interfaces to allow a mission specialist to work with small UAVs have either considered speech for mission scenarios where manual or visual interaction is not possible [12], speech in terms of improving speech understanding, not enabling audio communication [13], or speech acts as the framework for reasoning and problem-solving [14]. The two interfaces used in this paper to illustrate potential safety issues allow manual and visual interaction, as well as speech.

\section{EXERCISES}

The user interfaces were developed over a four year period starting in 2011, with funding as joint project between researchers and professional responders at Texas A\&M University starting in 2012. Development relied on monthly informal flights with feedback from responders from TEEX, five formal exercises with responders from participating agencies such as the Texas Commission on Environmental Quality, a set of controlled human-robot interaction experiments, plus one deployment to the 2014 SR530 Washington State Mudslides USA. The exercises and experiments were at the Disaster City ${ }^{\circledR}$ complex (Fig. 1) with the exception of a 2013 training event with South Carolina Task Force 1 at the South Carolina Fire Training Academy. Disaster City ${ }^{\circledR}$ is one of the training facilities used by TEEX to train fire rescue teams. Flight missions were primarily hazardous materials (hazmat) spills using the chemical train derailment prop, though an exercise in 2014 focused on the use of unmanned aerial systems for flooding and the creek/pond at Disaster City ${ }^{\mathrm{R}}$.

The chemical train derailment prop replicates a train carrying a variety of chemical tank cars. The train has derailed due to hitting a tractor-trailer carrying chemicals at a road crossing. The prop is challenging for responders because it is surrounded by trees blocking the view with binoculars; the trees also present challenges for UAS flights. The derailment is next to a gas pipeline that may or may not be ruptured due to the derailment and there is a nearby a oil tank that may be leaking. The responders must identify the types of chemicals being transported by the vehicles, either through reading the

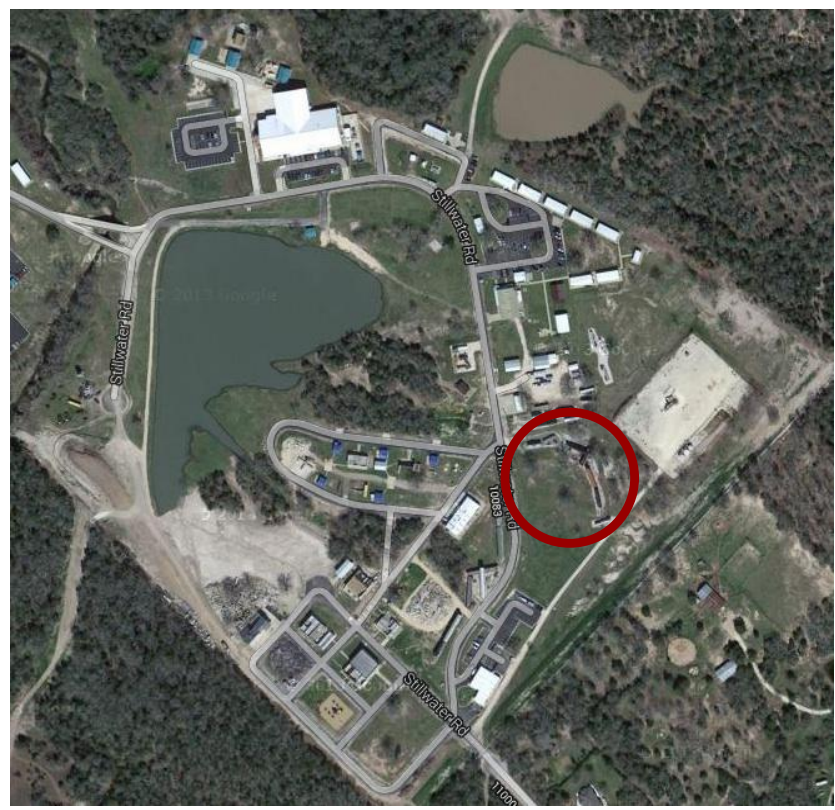

Fig. 1. Disaster City ${ }^{\circledR}$ complex with the chemical train car derailment circled.

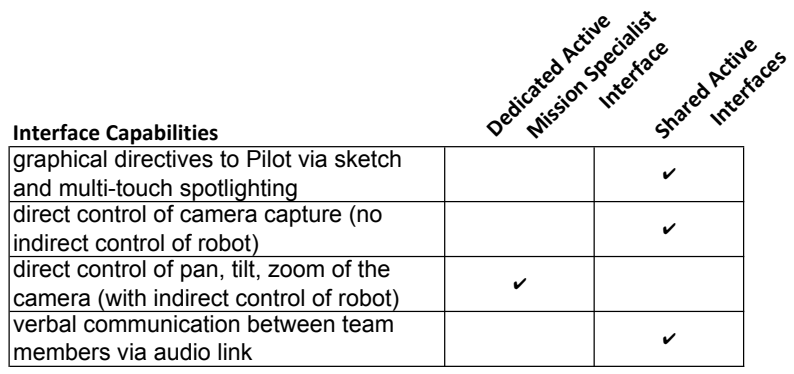

TABLE I

DIFFERENCE BETWEEN INTERFACES IN TERMS OF CAPABILITIES.

multi-colored placards on the cars or by inferring from the type of car. They must read the serial numbers on the car to confirm contents and identify leaks and if possible where the leaks are coming from and what is the best mitigation strategy. The responders should also identify any other relevant factors such as a waterway that might be polluted by the spill or a school that should be evacuated. Since the chemicals may be toxic or explosive, the responders would ideally do this from a distance without having to suit up in cumbersome personal protection gear.

\section{Sample Mission Specialist Interfaces}

When discussing the interfaces presented in this paper, a specific lexicon will be used and is based on the 
phrasing developed by Peschel et al. in [3]. Dedicated means an interface was specific to a role, e.g., a Pilot or Mission Specialist role. Interfaces can be active, allowing the user to directly interact with Pilot or the robot, or passive. The Dedicated Active Mission Specialist interface allows the Mission Specialist to actively control the camera but does not provide a mechanism for interacting with the Pilot other than verbal directives. The Shared Active interfaces are a set of interfaces that allow the Mission Specialist and Pilot to communicate with each other by sketching and spotlighting on the shared display of the robot's camera video. The capabilities of each interface are summarized in Table I and described in more detail below.

\section{A. DEDICATED ACTIVE MISSION SPECIALIST IN- TERFACE}

The Dedicated Active Mission Specialist Interface was created by Peschel [3]. The goal was to provide the Mission Specialist with a display tailored explicitly to that role, rather than simply mirroring the Pilot's display, while allowing the Mission Specialist and Pilot to maintain a visual common ground [15]. As can be seen in Fig. 2, the display consists of two windows. The larger primary window is the view from the payload camera; it is the same view as the Pilot's but it is stripped of any artifacts such as robot state, remaining battery life, etc., which was not considered important by the responders. The smaller window is a GIS view of the general area of the site and the dot and arrow shows where the sUAS is currently located and the direction it is facing. The interface allows the Mission Specialist to use multi-touch commands on the primary window to actively control the pan (swipe left or right to yaw the aircraft), tilt (swipe up or down), and zoom (pinch) of the camera and take high resolution pictures (button). Because the Mission Specialist can control the tilt and zoom, icons provide feedback. The interface also allows the Mission Specialist to capture the low resolution image on the primary window.

This interface has benefits for both the Mission Specialist and Pilot. For the Pilot, transferring control to the Mission Specialist helped achieve a "sterile cockpit"where the Pilot was not distracted by the Mission Specialist's verbal directives or even the occasional hand on their display. When the Mission Specialist spoke to the Pilot, they shared the same frame of reference avoiding problems with "whose left" seen in [16]. The Mission Specialists were more comfortable and empowered with this display over a mirrored display [17].

While the interface was preferred over a mirrored display by the Mission Specialists, it did present problems

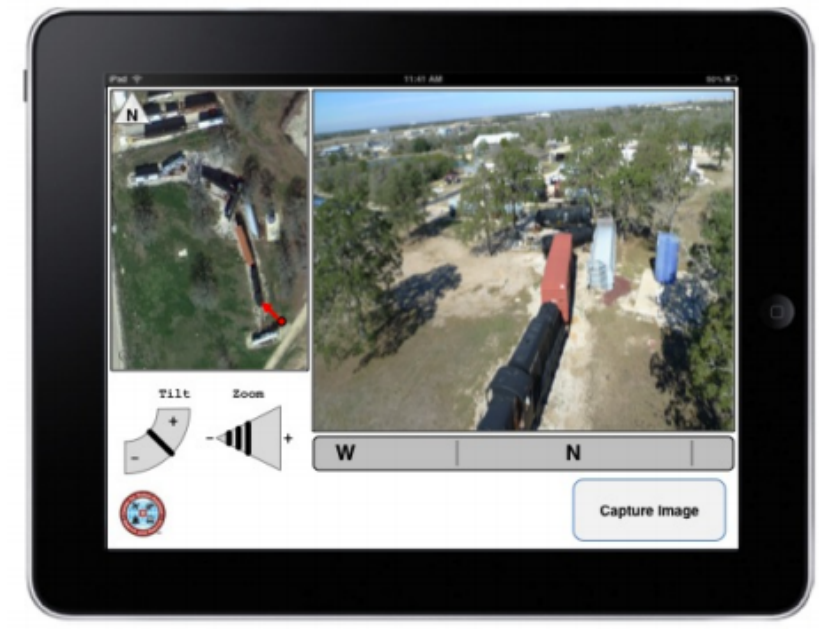

Fig. 2. Dedicated Mission Specialist Interface.

for the Pilot. These problems were due to the fact that the camera pan is accomplished by a UAS yaw, and because the Mission Specialist could pan, tilt, and zoom the camera during critical maneuvers such as take-off and landing and navigating in cluttered regions. Not only did these capabilities change the Pilot's view, but had the pan capability resulted in a yaw, depending on conditions, that yaw could result in a translation that led to a collision with a tree or structure at low altitudes. During experimentation with inspecting a chemical train derailment [17], the problem of unexpected camera and robot movement- even when instructed not to move the camera until the sUAS was on station- was significant enough that the software was modified to disable Mission Specialist control until enabled by the Pilot after the sUAS was on-station. The Pilot for the experiments also found the tendency for some Mission Specialists to alternate between direct verbal commands and control distracting and confusing. The flight team as a whole was concerned that the direct active interface risked the possibility of the Pilot losing situation awareness of the state of the robot in the environment.

\section{B. SHARED ACTIVE INTERFACES}

The Shared Active Mission Specialist Interface and corresponding Shared Active Pilot Interface were created by Henkel and Suarez [18] with two goals. The first goal was to extend the Dedicated Active Mission Specialist Interface to enable the Mission Specialist to communicate with the Pilot via sketch and spotlighting over the shared visual common ground. The communication was two-way, so the Pilot's interface was also modified. The second goal was to enable multiple Mission 
Specialists working from distributed locations to interact with the Pilot by creating a web-based interface viewable by a browser over the internet. The interface eliminated direct control of any function of the camera in order to prevent the problems with loss of situation awareness and control discussed in the previous subsection.

As shown in Fig. IV-B, the Shared Active Mission Specialist interface intentionally looks similar to the Dedicated Active Mission Specialist Interface. The secondary window with the contextual map is now larger as the icons for the camera tilt and zoom controls are no longer needed and the dot and arrow is replaced with with a larger dot and viewing angle indicator. The most significant change is that the primary window supports the user sketching and spotlighting. Fig. 3 shows the Pilot's complementary Shared Active Interface. The Pilot's interface sees the sketching and spotlighting from the Mission Specialist(s) and the Pilot can also sketch and spotlight. The Pilot can clear all screens of sketches thus clearing the Pilot's display. The Mission Specialist can only clear their sketches, thus communicating with the Pilot independently of any other users. The Mission Specialist presses a button to indicate when they want a high resolution photo taken and both the Mission Specialist and Pilot hears a recorded voice "photograph requested" as confirmation of the request. The window going black indicates the photo is being taken so no additional acknowledgment is needed. The Shared Active interfaces also were intended to have audio to allow team members to talk and sketch or spotlight at the same time to reinforce and clarify directives, though the links did not consistently work and were not tested with responders.

The benefits of the Shared Active interfaces are that they supported naturalistic team communication, were much faster to learn, and reduced the stress on the Pilot from uncontrolled movements of the platform or camera. The elimination of the direct control of the camera reduced the impact on the Pilot and reduced the responsibility of the Mission Specialist for being familiar with the robot. The addition of sketching and spotlighting was intuitive and responders who had used the Dedicated Active Mission Specialist Interface preferred the Shared Active even though they lost control. The sketch feature, where the user draws on the window, is typically used by Mission Specialists to circle and draw an arrow to indicate an object of interest and a desired angle of approach. The Mission Specialists will often write "done" when finished with the mission. The Pilot frequently uses the sketch feature to write notes to the Mission Specialists, e.g., "ready" for when the UAS

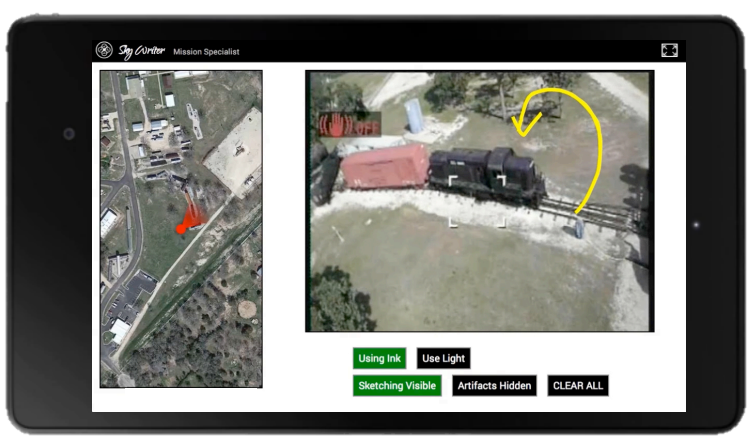

(a)

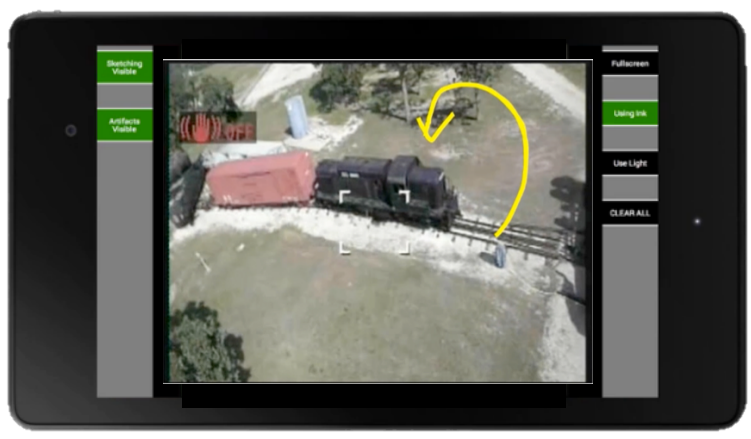

(b)

Fig. 3. Shared Active interfaces: a) Mission Specialist and b) Pilot Interface.

is on-station and "too much" when Mission Specialists individually or collectively begin to make too many demands. The spotlighting feature, where a user presses on the window until a moveable bright spot appears, has been used spontaneously by Mission Specialists for two functions. One common function is to hold the light at the edge of the image to indicate that the object of interest is offscreen and the camera or robot should move in that direction. The other function is to highlight an object that might be moving, or appear to be moving due to the image shaking, that they would like to approach or zoom in on; a sketch is static on the window and thus the object may move faster than the Mission Specialist can redraw.

While the Pilots and Mission Specialists preferred the Shared Active interfaces to the Dedicated Active Mission Specialist interface or mirror displays, problems did emerge. While team coordination between the Pilot and a single Mission Specialist was enhanced, team coordination with multiple Mission Specialists tend towards chaos. Multiple Mission Specialists often sketched and spotlighted independently, even for scenarios where they 
were designated as strictly observers, creating a highly cluttered Pilot's view that often resulted in the Pilot turning off all sketching and spotlighting. The spotlighting function works by darkening the screen except for the dot; while no flight problems were observed, darkening the screen does degrade the Pilot's view.

\section{SAFETY CONCERNS}

The two interfaces illustrate three distinct categories of safety concerns stemming from allowing distributed users to actively interact with the sUAS and Pilot: unsafe control regimes, loss of Pilot situation awareness, and increased Pilot stress.

\section{A. Unsafe Control Regimes}

The Dedicated Active Mission Specialist Interface combined with a sUAS platform without an independent gimbal for the camera posed two unsafe control regimes. As witnessed in the exercises, allowing the Mission Specialist to yaw the robot in order to pan the camera put the sUAS at risk of translating due to GPS error or a sudden gust of wind and thus at risk of hitting a tree or structure. Having two mission specialists trying to control the vehicle or a single camera would lead to directive "collisions" and if each were sending conflicting vehicle commands, unpredictable vehicle behavior could emerge.

\section{B. Loss of Pilot Situation Awareness}

The Dedicated Active Mission Specialist Interface increased the Mission Specialist's usability of the sUAS at the cost of the Pilot's situation awareness. The source of loss of situation awareness, following Endsley's definitions in [19], was due to the the Pilot's inability to control the viewpoint. When the Mission Specialist takes control of the camera, the Pilot has no means by which to perceive the movement of the platform relative to threats in the environment, e.g., loss of Level 1 SA (Perception). It can be difficult to tell if the robot is drifting or changing altitude from an arbitrary viewing angle. Without being able to actively look around and update the understanding of the environment, the Pilot also loses the ability to generate Level 2 SA (Comprehension) and Level $3 \mathrm{SA}$ (Projection) needed to project possible problems, emergency landing strategies, etc.

Under the Dedicated Active Mission Specialist Interface, the Pilot can take back control of the camera if an anomalous condition occurs, but a smooth transfer of control from the Mission Specialist to the Pilot is not

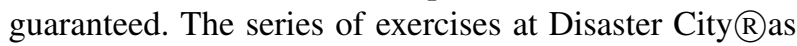
well as structural inspection flights in the aftermath of Hurricane Katrina found that when the Pilot had to take back control to move the vehicle or return to home, it was easy to become disoriented because of the tendency of the Mission Specialists to leave the camera zoomed in and at an unatural angle for flying.

\section{Increased Pilot Stress}

The Shared Active Interfaces avoid explicitly unsafe control regimes and eliminate the loss of the Pilot's ability to maintain situation awareness but it increases the Pilot's stress due to increased cognitive load. Four sources of increased cognitive load have been observed with the Shared Active Interfaces during exercises; these are presented in order of severity of impact:

Graphical and audio communication from multiple Mission Specialists. Multiple Mission Specialists avoided extensive or contradictory sketching, probably because they could easily see how the imagery was being obscured. However, there appeared to be no natural disincentive to use spotlighting, which leads to overdarkening of the screen. The most significant instance of the multi-user directive collisions occurred when three users were all using spotlight to direct the pilot to different places. As can be seen in Fig. 4, the pilot simply wrote "too much" and then began ignoring all input from the Mission Specialists. The audio link implementation for multiple users did not work consistently, but we speculate that this will be a source of communication conflict from multiple Mission Specialists.

Increased workload due to performing some Mission Specialist functions. The Pilot was flying, yet had to take photos.

Graphical communication mechanisms degrading visual display. Spotlighting was a popular choice by Mission Specialists but it requires darkening the screen slightly. No instances of Pilot error (or frustration) were observed when a single Mission Specialist used the spotlight during exercises.

Distracting audio communication. Irrelevant or frequent audio communication has the potential for distracting and increasing the cognitive load of the Pilot; however, responders are trained in radio communications and their verbal commands were effective in amplifying the graphical indicators. Instead, the quality of the audio link was observed to be a distraction, with poor quality producing visible signs of frustration by the Pilot.

\section{RECOMMENDATIONS}

The experiences with the two different interfaces suggest that ultimately it is desirable to allow the Mission Specialists to be able to perform their role rather than increasing the Pilot's cognitive workload by absorbing the camera operations and deconflicting multiple requests. 


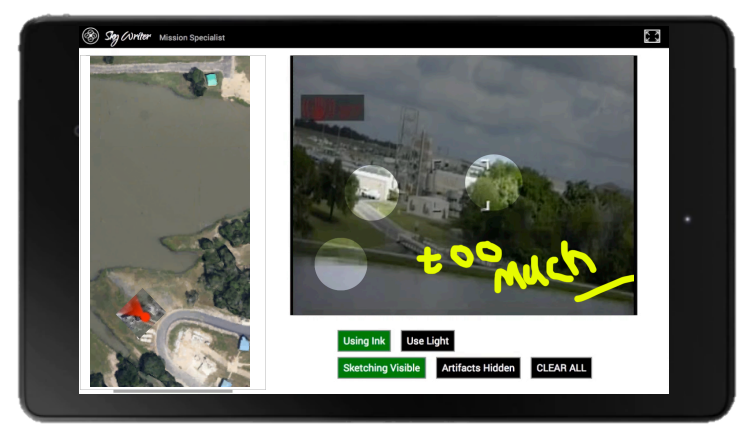

Fig. 4. Shared Active Mission Specialist Interface displaying multispotlight points and a pilot sketch reflecting "too much" information.

Ideally a combination of the two Active interfaces would be created. The improved interface would be Shared enabling team coordination; Mission Specialists to use sketch, spotlight, and audio to communicate deconflicted platform directives to the Pilot, providing team members with a highly naturalistic mechanism for teamwork. The Pilot's improved Shared Active Interface would support the maintenance of situation awareness. The Mission Specialist's improved Shared Active Interface would be similar to the Dedicated Active interface, allowing the Mission Specialist to direct control the payload but eliminating entering unsafe control regimes.

We offer five recommendations on how reach the desired interaction and interface for distributed users while promoting safety:

Separate the payload camera from the platform, giving the Pilot a dedicated "pilot-cam" and the Mission Specialist a fully gimbaled payload to control that does not require yawing the robot. This would enable the Mission Specialist to directly perform camera operations without turning the robot platform. The "pilot-cam" would allow the Pilot to actively maintain situation awareness at all times without having to be subordinate to the Mission Specialist. This would introduce a different style of team coordination as the Mission Specialist (or artificial intelligence) would have to compensate for the platform's movements to keep the camera payload on track.

A separate, fully gimbaled payload is not a new idea: the iSENSYS IP3 sUAS used at Hurricane Katrina and the Berkman Plaza collapse was equipped with one [1]. The Pilot for those flights preferred this to the "pan=yaw" configuration and it did not impact the Mission Specialist. Separating the cameras from the platform controls has appeared in commercial sUAS targeted for videography, but these manufacturers typically assumes the Pilot will look at the vehicle while flying, aka heads-up or taking an exocentric viewpoint. Flying heads-up limits the effective distance for using the robot for disaster situations because it makes it extremely hard to judge position relative to structures.

Use artificial intelligence for conflict resolution between competing directives from multiple Mission Specialists. Conflict resolution algorithms for planning and resource scheduling are common in artificial intelligence and could be applied to reduce Pilot cognitive load from having to resolve competing requests and reduce distraction from listening to or seeing the requests. Algorithms would rely on the rules such as "share time equally between Mission Specialists" or to give priority based on the chain-of-command. Alternatively, scheduling algorithms could attempt to aggregate the directives, for example minimizing the number of vehicle movements or determining the direction of interest to the most participants.

Allow the Pilot or a software agent, to turn off Mission Specialist control or communication. A partial alternative to redesign of sUAS to have a completely independent payload is to bar the Mission Specialist from exercising control at the wrong times. One solution is a button on the Pilot's interface, which requires the Pilot to take action. In some cases, the Pilot may not be able to disable Mission Specialist control and deal with the current unexpected situation created by the Mission Specialist. A more attractive solution is to create intelligent software to infer when control by a Mission Specialist is inappropriate, such as take off and landing, returning home, or when the battery level is too low to continue a flight.

Use multi-modal warnings in Pilot interfaces rather than relying on visual cues. The preference for the Active Shared Interfaces highlights the potential to overuse the visual channel of communication. The Pilot's interface is already subject to darkening and clutter from sketches. However, the audio channel is available and warnings can override any verbal conversation from Mission Specialists.

Add guarded motion to prevent collisions. While allowing the Pilot (or robot) to selectively bar Mission Specialist control helps with overcoming the safety risks from the "pan=yaw" style of payload, it is not a complete solution. Even though the Mission Specialist is legitimately working, they can introduce unsafe movements. Despite GPS and IMU advances, there is still a tendency for platforms to drift off station, especially when yawing. As noted in [20], artificial intelligence could be used to to build at least a coarse understanding 
of the environment and react to obstacles such as trees and buildings.

\section{CONCLUSIONS}

Our experience indicates that remotely-viewable interfaces for distributed users (Mission Specialists) are desirable for safety, security, and rescue sUAVs. These interfaces allow one or more experts who are unfamiliar with the sUAS focus on the mission and allows them to work over the internet, coordinating with the Pilot. However, our experiences also indicate that Active interfaces that allow the Mission Specialist to actively control the robot or engage the Pilot pose safety risks. These risks can be mitigated with both hardware and software changes. The hardware can reduce risk by moving away from sUAS where the payload camera is the pilot cam or the payload camera requires the platform to yaw in order to effect a camera pan. Artificial intelligence can reduce risk in three ways: it can manage conflict resolution if multiple Mission Specialists give the Pilot competing directives, provide multi-modal warnings to the Pilot rather than use visual channels which are being used for situation awareness and team coordination, and for providing guarded motion.

\section{ACKNOWLEDGMENTS}

This work was conducted as part of a NSF Graduate Research Fellowship and was supported in part by NSF Grant IIS-1143713 EAGER: Shared Visual Common Ground in Human-Robot Interaction for Small Unmanned Aerial Systems and used the RESPOND-R mobile, distributed test instrument, NSF Grant CNS0923203. The authors are grateful to the Texas A\&M Engineering Extension Service for the facilities, expertise, helpful discussions and insights, and to Zack Henkel, Dr. Joshua Peschel, and Jesus Suarez.

\section{REFERENCES}

[1] R. R. Murphy, Disaster Robotics. Cambridge, MA: MIT Press, 2014.

[2] — "The 100:100 challenge for computing in rescue robotics," in IEEE International Symposium on Safety Security Rescue Robots, 2011, pp. 72 - 75.

[3] J. Peschel, B. A. Duncan, and R. Murphy, "Exploratory results for a mission specialist interface in micro unmanned aerial systems," in 2012 International Conference on Collaboration Technologies and Systems (CTS), 2012, pp. $131-140$.

[4] J. Peschel and R. R. Murphy, "On the human-computer interaction of unmanned aerial system mission specialists," IEEE Transactions on Human-Machine Systems, vol. 43, no. 1, pp. 53-62, 2013.

[5] T. J. Gledhill, E. Mercer, and M. A. Goodrich, "Modeling uass for role fusion and human machine interface optimization," in Systems, Man, and Cybernetics (SMC), 2013 IEEE International Conference on, 2013, pp. 1929-1937.
[6] D. Cavett, M. Coker, R. Jimenez, and B. Yaacoubi, "Humancomputer interface for control of unmanned aerial vehicles," in Systems and Information Engineering Design Symposium, 2007. SIEDS 2007. IEEE, 2007, pp. 1-6.

[7] T. Pond, B. Webster, J. Machuca, J. Colombi, M. Miller, and R. Gibb, "Allocation of communications to reduce mental workload," Procedia Computer Science, vol. 8, no. 0, pp. 242-248, 2012.

[8] K. Pratt, R. Murphy, S. Stover, and C. Griffin, "Conops and autonomy rcommendations for vtol suass based on hurricane katrina operations," Journal of Field Robotics, vol. 26, no. 8, pp. 636-650, 2009

[9] D. Cummmings, S. Fymat, and T. Hammond, "Reddog: a smart sketch interface for autonomous aerial systems," pp. 21-28, 2012.

[10] C. Guo, J. E. Young, and E. Sharlin, "Touch and toys: new techniques for interaction with a remote group of robots," pp. 491-500, 2009.

[11] F. Jourde, Y. Laurillau, and L. Nigay, "Comm notation for specifying collaborative and multimodal interactive systems," pp. 125-134, 2010.

[12] D. F. Allinger, C. Strauss, and D. Kwon, "Applications of speech technology to unmanned vehicles," in Digital Avionics Systems, 2001. DASC. 20th Conference, vol. 1, 2001, pp. 5B4/1-5B4/9 vol.1.

[13] J. Ferreiros, R. San-Segundo, R. Barra, and V. Pérez, "Increasing robustness, reliability and ergonomics in speech interfaces for aerial control systems," Aerospace Science and Technology, vol. 13, no. 8, pp. 423-430, 2009.

[14] P. Doherty and F. Heintz, "A delegation-based cooperative robotic framework," in Robotics and Biomimetics (ROBIO), 2011 IEEE International Conference on, 2011, pp. 2955-2962.

[15] J. Burke and R. Murphy, "Rsvp: An investigation of remote shared visual presence as common ground for human-robot teams," in ACM/IEEE Human-Robot Interaction, 2007, 22

[16] M. van Zomeren, J. M. Peschel, T. Mann, G. Knezek, J. Doebbler, J. J. Davis, T. A. Hammond, A. H. J. Oomes, and R. R. Murphy, "Human-robot interaction observations from a protostudy using suavs for structural inspection." in HRI, 2009, pp. 235-236.

[17] J. M. Peschel, "Mission specialist human-robot interaction in micro unmanned aerial systems," Ph.D. dissertation, 2012.

[18] Z. Henkel, J. Suarez, B. Duncan, and R. Murphy, "Sky writer: Sketch-based collaboration for uav pilots and mission specialists," in ACM/IEEE International Conference on Human-Robot Interaction, 2014

[19] M. R. Endsley, "Toward a theory of situation awareness in dynamic systems," Human Factors: The Journal of the Human Factors and Ergonomics Society, vol. 37, no. 1, pp. 32-64, 1995. [Online]. Available: http://hfs.sagepub.com/content/37/1/32.abstract

[20] K. Pratt and R. R. Murphy, "Protection form human error: Guarded motion methodologies for mobile robots," IEEE Robotics and Automation Magazine, vol. 19, no. 4, pp. 36-47, 2012, subbmission number 10-0036, resubmitted 7/30/11. 